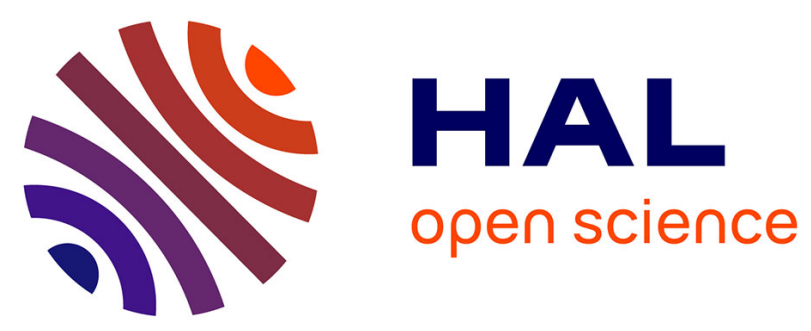

\title{
Meeting report Montpellier Infectious Diseases (MID), 2nd Annual Meeting (2012)
}

P Bastien, C Braun-Breton, N Chazal, C Devaux, V Foulongne, M Frank, Jean-François Hernandez, L Kremer, M Lebrun, V Le Moing, et al.

\section{- To cite this version:}

P Bastien, C Braun-Breton, N Chazal, C Devaux, V Foulongne, et al.. Meeting report Montpellier Infectious Diseases (MID), 2nd Annual Meeting (2012). Infection, Genetics and Evolution, 2013, 16, pp.450-454. 10.1016/j.meegid.2013.01.019 . hal-02149417

\section{HAL Id: hal-02149417 https://hal.science/hal-02149417}

Submitted on 7 Jun 2019

HAL is a multi-disciplinary open access archive for the deposit and dissemination of scientific research documents, whether they are published or not. The documents may come from teaching and research institutions in France or abroad, or from public or private research centers.
L'archive ouverte pluridisciplinaire HAL, est destinée au dépôt et à la diffusion de documents scientifiques de niveau recherche, publiés ou non, émanant des établissements d'enseignement et de recherche français ou étrangers, des laboratoires publics ou privés. 


\section{Meeting report}

\section{Montpellier Infectious Diseases (MID), 2nd Annual Meeting (2012)}

\section{Introduction}

Discovery of antibiotics, antiviral and anti-parasitic compounds during the last 70 years have resulted in great hope to definitely overcome most infectious diseases. However, today, like centuries ago, millions of people die each year from infectious diseases and this sad evidence confronts us with our fear from illness and death. In recent decades, the issue of emerging and re-emerging infectious diseases has become an increasingly important area of concern in public health/global health. The rapid spread of infectious agents is inseparable from the widespread existence of pathogens in wildlife reservoirs, their transfer to humans and the continuous increase in travel activity (the "One world one health" concept), contributing to the rapid spread of pathogens all over the world. Moreover, genetic changes result in adaptation of the infectious agents to new ecological niches and sometimes in acquisition of drug resistance, allowing them to escape the armamentarium available to clinicians today. It is therefore crucial to reinforce discussions between biologists, chemists, biophysicists and physicians to identify new therapeutic avenues in the control of epidemics that threaten human societies.

For the second consecutive year, teams of the network "Montpellier Infectious Diseases" held their annual meeting. The ambition of the MID conference is to bring together teams working on infectious diseases, in order to promote interconnections and collaborations between research projects. In addition MID should act as a think tank including the interactions with invited speakers, to collectively identify research areas of priority deserving focus of the efforts of the MID consortium. Ultimately, the objective is to strengthen or bring out areas of scientific excellence for the site of Montpellier and to develop them in a coordinated way, thereby responding to major international challenges regarding infectious diseases.

As last year, about 200 people attended this meeting. Since the 2011 meeting was focused on host-pathogen interaction and pathophysiology (vectors and reservoirs; immunopathology and infections and cancers; Mediterranean and Southern regions; intracellular life; emergence and resistance to antibiotics; new therapeutic targets), it was decided that the 2012 meeting, held on November $29^{\text {th }}$ and $30^{\text {th }}, 2012$, should focus on the cooperation between medical and chemical sciences interdisciplinary approaches to fight again drug resistant pathogens.

For example, during the past 50 years a great number of antiviral drugs have been developed and are now licensed for clinical use in the treatment of viral infection caused by human immunodeficiency virus, herpes simplex virus, cytomegalovirus, varicella zoster virus, hepatitis B and C viruses and influenza viruses. Recent knowledge about the natural history and pathogenesis of viral disease has allowed the design of a large panel of new antiviral compounds. Most of the major successful stories in antiviral drugs developments result from 
collaborative efforts between a number of experts, including physicians, academic and industrial researchers in numerous fields from virology, cell biology, to biochemistry. The Montpellier Infectious Diseases was the opportunity to confirm and illustrate the local presence of similar collaborative network and to highlight its great value in the fight against virus, bacteria and parasites.

\section{Session Summaries}

\subsection{Virology session}

Christian Devaux (CNRS, UMR 5236, UM1,UM2) and Vincent Foulongne (INSERM U1058, CHUR), Chairmen

\subsubsection{Nucleoside analogues: the core of the antiviral armamentarium}

The key role of nucleoside analogue was illustrated by a fascinating success story "from the bench to patient", presented by Pr Gilles Gosselin (Idenix) who described the development of a new hepatitis B virus (HBV) inhibitor of the viral polymerase, the telbuvidine. Viral polymerase including DNA polymerase, RNA polymerase and reverse transcriptase are essential for viral replication and are, as such, obvious targets for viral inhibition and treatment. Nucleoside analogues were the first viral polymerase inhibitors that have shown a clinical efficiency and are now extensively used as DNA polymerase inhibitors in the treatment of Herpesviridae infections or as reverse transcriptase inhibitors in both HIV and HBV infections. Hepatitis B is a global health concern with up to 350 million of chronically infected people in the world leading to near a million deaths a year. Hepatitis B virus is a DNA virus harboring a reverse polymerase that could be a target for nucleoside analogues, and few drugs are currently available such as lamivudine, adefovir or entecavir. Natural nucleosides present a D-configuration and, during decades, it was thought that only D-nucleoside enantiomer analogues could exhibit a biological activity. The L-nucleoside enantiomers are mirror images (non superimposable) of their respective natural Denantiomers. The first L-nucleoside with a significant biological activity was the lamivudine (3TC) that have opened the way for the development of alternative L-nucleoside analogue candidates. Among them was the telbuvidine (LdT), a very simple chemical structure as it is the mirror image of the thymidine (dT), a natural nucleoside. The telbuvidine developed by Idenix in a close partnership with Novartis had proven a good specificity and selectivity against HBV. Next, a phase II clinical trials demonstrated a high degree of viral suppression, and an international phase III clinical trial named the "GLOBE study" confirmed the efficiency of telbuvidine leading to the marketing approval by both the FDA and the European medicine agency. The telbuvidine is currently worldwide used as an oral, once daily regimen for the treatment of chronic hepatitis B under the commercial names of Tyzeka ${ }^{\circledR}$ and Sebivo®.

Another talk concerned nucleoside phosphonate analogues. Nucleoside analogues are prodrugs that need to be phosphorylated to their 5'-triphosphate forms. These later can then interact with the viral polymerase as competitive inhibitor or as alternate substrate for the viral enzyme thus preventing viral nucleic acid chain elongation. In this process, the initial 
phosphorylation of the nucleoside analogue to its 5'-monophosphate is considered as the rate limiting step towards the formation of the active 5'-triphosphate metabolite. Attempts to overcome that limiting step are illustrated by the designed of stable 5'-mononucleotide phosphate. Such compounds are nowadays considered as a potent class of antiviral agents illustrated by the HIV reverse transcriptase inhibitor, tenofovir or the cytomegalovirus DNA polymerase inhibitor, cidofovir. Within the cells, they must be metabolized to their corresponding diphosphate forms to exert their biological activity. In this process, the first phosphorylation step, catalyzed by nucleoside monophosphate kinases (NMP kinases), has been proposed as a bottleneck. In his presentation, Prof. Christian Périgaud (Institut Max Mousseron, UM2) has reported the synthesis of series of ribonucleoside phosphonate derivatives isosteric to 5'-mononucleotides and displaying different degrees of flexibility within the 5',6'-C-C-bond as well as different polarities, through the introduction of hydroxyl groups. The influence of these modifications on their capacity to act as substrates for appropriate human NMP kinases, involved in nucleic acids metabolism, has been investigated and results showed that a low flexibility as well as absence of hydroxyl groups within the ribose-phosphorus architecture are both critical for efficient phosphotransfer.

The lecture from Prof. Christophe Mathé (Institut Max Mousseron, UM2) was a nice review and conclusion of previous talks related to the use of nucleoside analogues as antiviral drugs. He presented an overview of the nucleoside covering area such as structure, enzymatic targets and the different strategies for their synthesis and chemical improvement. He summarized the various strategies that chemists could use to optimize the nucleoside analogue activities; it could be achieved through modifications in nucleoside or nucleoside phosphate design allowing them to act as better substrates for the cellular kinase. These modifications could involve the structure of both nucleotides or sugars and regarding the later it has been shown that their multiple conformation capabilities may impact the property of the nucleoside analogue. This was illustrated by the model of nucleosides analogue designed with sugars blocked by propyl residues in a given conformation

\subsubsection{Viral physiopathology and clues for antiviral targets}

An extensive knowledge of viral physiopathology is likely to trigger researcher's interest towards clues for antiviral target candidate and this was suggested in the talk of Doctor Bruno Beaumelle (CPBS, UMR5236, CNRS,UM1,UM2). The role of HIV-1 Tat as transcriptional regulator is well known but Bruno Beaumelle has investigated the role of Tat as a likely virulence factor of HIV-1 introducing the original idea of Tat as a "viral toxin". HIV-1 multiplication is restricted in macrophages. Nevertheless, the functional capacity of phagocytes from HIV-1 infected patients is impaired, allowing the multiplication of several opportunistic pathogens that are a major source of morbidity and mortality during AIDS. A secreted HIV-1 virulence factor could be responsible for the phagocytic defect of uninfected macrophages. A strong candidate is the HIV-1 Tat protein that is massively secreted by HIV-1 infected cells. This unconventional secretion is allowed by the very tight binding of Tat to phosphatidylinositol (4,5)bisphosphate (PIP2). Secreted Tat can then enter uninfected cells and severely interfere with phagocytosis. The molecular mechanism of this inhibition requires 
interaction of Tat with intracellular proteins. The data presented at the meeting indicated that circulating Tat can enter cells and thereby can be responsible for the phagocytic defects observed in uninfected phagocytes following HIV-1 infection. Extracellular Tat thus seems to be a key HIV-1 virulence factor that enables the development of opportunistic infections.

Next, a nice presentation by Landry Charlier (Max Mousseron Institute, UMR5247) gave us the opportunity to revisit, using molecular modeling approach, the mechanism and dogma of membrane anchoring of HIV-1 matrix protein before budding of virions. During HIV assembly in infected host cells, the interaction of the N-terminus Matrix (MA) domain of the viral Gag protein with the plasma membrane lipid PI(4,5)P2 appears as the first crucial step for particle assembly. This model is reinforced by recent lipidomic analysis of the viral envelop, showing an enrichment in $\mathrm{PI}(4,5) \mathrm{P} 2$. It was previously suggested that this anchoring might require a 'Myristoyl switch' i.e. the PI(4,5)P2 binding to MA occurs in an allosteric site that could promote Myristate exposure.It was also shown that the 2'-acyl chain (usually unsaturated) was able to flip into an hydrophobic pocket of MA, allowing therefore the complex to partition into pre-existing lipid microdomains, namely rafts2. Starting with the NMR structure of the Myristoylated HIV-1 MA protein, we performed multiple coarse grained molecular dynamics simulations to simulate the anchoring of the HIV-1 MA protein to a PI(4,5)P2 containing membrane model. Quite surprisingly, it was observed that the Myristoyl group could easily unbind from its initial hydrophobic pocket in MA thus allowing spontaneous anchoring of the protein to the membrane. More importantly, it was observed that after having found a stable orientation, the protein was able to confine PI(4,5)P2 all around its molecular surface with a preferential binding site in the same regions as that described by NMR data. But, on the contrary to what NMR showed, no flipping of the 2'acyl chain of the PI(4,5)P2 was observed. The acyl chains stayed within the hydrophobic core of the lipid membrane. Our model therefore suggested that the protein itself, after membrane anchoring, could be able to confine PI(4,5)P2, without any requirement of pre-existing domains, as suggested in Kerviel and coll. at CPBS. Moreover, estimation of the required energetic cost for myristoyl release suggested that no Myristoyl switch mechanism would be necessary.

Finally, a speaker addressed the question of the viral RNA capping machinery as target for antiviral drugs. Most viruses modify their genomic and m-RNA 5' ends with addition of an RNA cap. This allows a more efficient m-RNA translation, limits the degradation by cellular exonucleases and avoids its recognition as foreign RNA by host cells. The viral RNA capping machinery deciphers a high diversity of mechanisms, partners and pathways, and viral capping enzymes could be the targets of novel highly efficient and selective drugs. In her presentation, Dr. Françoise Debart has focused the need for the development of model that could allow the studies of viral capping enzyme inhibitors. Such model could be achieved by the production of stable capped RNA. She presented an original synthesis way for the production of large amount of capped RNA that combines solid support synthesis using the 2'-O-PivOM technology followed by a link through a 5' triphosphate to a guanosine, as previously described for the production RNA 5' triphosphate. RNAs are then released from both protection and support to acts as substrates in an enzymatic 
methylation reaction. Recovered RNAs are of enough quantity and quality to allow subsequent biochemical and structural analysis of their interactions with enzymes of the viral capping pathways.

\subsubsection{Field antiviral drug use, prevention of HIV-1 mother to child transmission through breastfeeding}

of Human Immunodeficiency Virus type 1 (HIV-1) mother to child transmission (MTCT) have beneficiated of advances in HIV-1 treatment of pregnant women eligible for highly active antiretroviral therapy (HAART) with a significant decrease of perinatal transmission rates. However, a shift to HIV-1 postnatal transmission is now observed with breastfeeding transmission responsible for near half of the new HIV-1 paediatric infections acquired yearly. Since, breastfeeding remains the most benefic feeding solution for babies in low resource countries, the prevention of HIV-1 transmission through breast milk is a challenging issue in African settings that was addressed by Prof. Philippe Van De Perre (Inserm U1058) in his presentation. HIV-1 transmission risk factors for breast fed infant include breast feeding duration, occurrence of mammary gland inflammation and mother high viral load in both plasma and breast milk. Since HIV-1 viral load in plasma is the major identified risk of transmission, recommendations for prevention of MTCT (PMTCT) rely on antiretroviral use. PMTCT guidelines include initiation or prolongation of treatment during both gestation and lactation for HAART eligible women and either daily AZT monotherapy until delivery switched by daily peri exposure prophylaxis (PreP) with nevirapine in newborn for the duration of breast milk exposure (option A) or early triple antiretroviral prophylaxis during pregnancy continued until one week after end of breastfeeding for women not eligible for HAART (option B). These two options are complementary but could presently not be combined. Randomised clinical trials demonstrated a relatively low efficacy of option B for preventing breastfeeding HIV transmission (52\% efficacy in the Kesho Bora trial). A recent study from Prof. Philippe Van De Perre lab have shown that activated CD4+ T lymphocytes producing intracellular HIV-1 RNA can be found in the breast milk of HIV-1 infected women taking ART (undetectable HIV-1 RNA in plasma and milk). These HIV-1 RNA cellassociated reservoirs are plausible candidates for cell-to-cell transfer of the virus. Therefore prevention of HIV-1 transmission by breastfeeding should be effective on both ongoing cycle of replication and on virus released from stable reservoirs that become productive in the mammary gland. The ANRS 12174 trial (ClinicalTrials.gov identifier: NCT00640263) is presently evaluating the efficacy of option A administered for 12 months. It compares the efficacy and safety of prolonged infant PreP with lopinavir/ritonavir versus lamivudine to prevent HIV-1 transmission through breast milk in children born to HIV-1-infected mothers not eligible for HAART and having benefited from perinatal ART regimens. The study has now completed enrolment in the four African countries: Burkina Faso, Uganda, Zambia and South Africa, and preliminary results show a low rate of residual transmission and strongly suggest that infant PreP is a valid strategy that could contribute to the elimination of HIV-1 transmission by breastfeeding. However, the adherence to a twice-daily oral regimen is an issue for many women. An injectable drug that can be given to the baby on a limited number 
of injections during the course of breastfeeding under direct supervision of a health care worker would mean a considerable alleviation of adherence issues and a much better protection for the infants. The novel diarylpyrimidine analogue Rilpivirine, a potent nucleoside reverse transcriptase inhibitor (NNRTI) active against wild type and NNRTIresistant HIV-1 strains is available as nanosuspension for long-acting injectable formulation. Preclinical studies in rats and dogs showed that the drug is well tolerated and results in stable plasma concentrations profiles for more than six weeks. The drug seems to be uptaken by macrophages and concentrated at very high level in lymph nodes and lymphoid tissues. Satisfactory phase I/II trials in human adult volunteers have been reported. Long acting Rilpivirine is an obvious candidate for clinical evaluation in HIV-1 exposed breastfed infants as an infant PreP with improved adherence.

\section{$2.2 \quad$ Bacteriology session}

\section{Laurent Kremer (CNRS, UMR 5235, UM2) and Jean-Paul Leonetti (Deinove), Chairmen}

\subsubsection{Mechanisms of virulence and novel drug developments}

This session, comprising 7 presentations, were mostly focusing on different mechanisms used by several pathogenic bacteria to regulate virulence determinants as well as mechanisms responsible for antibiotic resistance. Special emphasis on how to target virulence factors for the future development of new classes of antimicrobials has also been covered by several presentations. The keynote opening conference by B. Felden (INSERM U835, University Rennes 1) illustrated the new and unexpected role of ribonucleic acids in the physiology of Staphylococcus aureus, including environmental adaptation and metabolic regulations, and how these small regulatory RNAs participate in the control of $S$. aureus virulence. In addition, it appeared that many of the 100 small RNAs identified in this bacterium could represent attractive targets to be exploited for the development of new antibiotics and/or diagnostic tools. The second presentation by C. Lionne (CPBS, UMR 5236) was related to the enzymatic mechanism of resistance of aminoglycoside-based antibiotics. Aminoglycoside phosphotranferases are important bacterial enzymes able to inactivate aminoglycosides by $O$ phosphorylation, a chemical modification that render bacteria resistant to this class of antimicrobials. Of interest was the description of the kinetics of aminoglycoside phosphotransferase (3')-IIIa that identified an ADP-enzyme complex as the main transient intermediate during the reaction. Therefore, an attractive strategy to prevent phosphorylation of aminoglycosides would be to develop inhibitors that interact specifically with the ADPenzyme complex. M. Lopez (CPBS, UMR 5236) illustrated how a small molecule inhibiting a virulence factor could represent a strategy aimed to prevent replication of various intracellular pathogens. The histidinol dehydogenase (HDH) participating in histidine metabolism is essential for Brucella suis, the causative agent of human brucellosis, and is also required for the intramacrophagic growth of Mycobacterium tuberculosis, the causative agent of tuberculosis. A combination of chemical studies together with molecular modeling led to the discovery of inhibitors interfering with the active site and the NAD binding pocket of HDH. These promising results are currently pursued by the screening of new libraries of 
compounds. Therefore, targeting HDH may lead to the discovery of new antibiotics targeting these two important human pathogens. However, M. tuberculosis appears extremely difficult to eradicate, not only because of the emergence of multi-drug and extremely-drug resistant strains but also because the bacterium can persist for years or even decades in the infected host. A non-replicative dormant form can survive within infected granulomas where most drugs are inoperant. In addition, dormant tubercle bacilli become phenotypically resistant to most antitubercular drugs. In this context, D. Domurado (Institut Max Mousseron, UM1) has synthesized a pro-drug based on ibuprofen which has shown potent activity in vitro in a nutrient starvation model of $M$. tuberculosis that mimics persistence. This example is an interesting illustration demonstrating that a 40-years old anti-inflammatory molecule exhibits a potent and totally unexpected activity against dormant bacilli. As an alternative to conventional antibiotics, human defensins HNPS 1-3, which are important molecules of the innate immunity, are also active against many Gram positive and Gram negative bacteria at a low concentration, making them excellent candidates for the development of new antiinfective drugs. L. Voissier (Etablissement Français du Sang) presented an original approach to produce HNPs 1-3 based on the purification of $\square$-defensins from neutrophils retained on the fractionation filters followed by an immunoprecipitation using paramagnetic nanobeads. This process leads to pure HNP preparations highly active against various bacteria, thus offering new candidates for effective weapons against multiresistant bacteria.

\subsubsection{New insight into the detection of bacteria and applications in diagnosis}

As mentioned above, the excessive use of antibiotics has led to the emergence of resistant strains, thus prompting to the search for new anti-infective compounds and a rapid identification of their antibacterial efficiencies. H. Cottet (Institut des Biomolécules Max Mousseron, UMR 5247, UM2) presented a new technology based on capillary electrophoresis to study the antibacterial activity and the interactions between antibacterial compounds and bacteria. This technology could speed up the antibiogram determination. F. Veas (IRD, ApoH-Technologies) pointed to the fact that most standard diagnosis approaches are poor in performances and/or generating false negative diagnostics. This is particularly the case for biological samples containing low amounts of microorganisms, an issue that can be overcome by increasing the pathogen detection. This can be achieved by a pre-treatment of complex biological samples with Apolipoprotein $\mathrm{H}(\mathrm{ApoH})$, a protein able to capture a large spectrum of microorganisms. ApoH-coated solid supports allow to increase their concentrations dramatically, thus improving their subsequent detection using standard qPCR, ELISA or cultivation, and enhancing diagnostic performances.

\subsection{Parasitology session}

Catherine Braun-Breton (CNRS,UMR5235,UM2) and Patrick Bastien (IRD), Chair

The Parasitology session presented a large diversity of approaches aiming directly or indirectly to the discovery of new drugs, novel drug targets or specific pathways potentially useful for drug development against parasitic diseases. In her introductory conference, 
Dominique Mazier (UMR S945 INSERM-UPMC) brilliantly illustrated these concepts by the recent development in her group of an in vitro model of the pre-erythrocytic cycle of Plasmodium cynomolgi, allowing the study of its 'dormant' forms or hypnozoites. This model is being used for characterising the molecular mechanisms underlying 'dormance' and reactivation, which appear highly dependent upon genetics/epigenetics of the parasite .

Genome sequencing, now achieved for most protozoan parasites, has confirmed the complexity and specificity of the biological/biochemical processes present in these 'divergent' eukaryotes. With $>50 \%$ of the proteins yet totally unknown, they offer wide perspectives for investigation. Thus, in his presentation of the recently finished genome sequencing of the emerging human pathogen Babesia microti, Emmanuel Cornillot (Laboratoire de Biologie Cellulaire, EA4558, UM1) could identify several targets suitable for diagnosis and treatment of human babesiosis. He showed that B. microti has the minimal metabolic requirement for intraerythrocytic protozoan parasitism, and that two lateral transfer events with significant metabolic implications occurred during the evolution of this parasite.

With the aim of helping researchers to improve the functional annotation of parasite proteins, Laurent Bréhélin and colleagues (LIRMM, UMR 5506 CNRS- UM2) developed the EuroPathDomains database: a divergent domain database for ten eukaryotic pathogens. Indeed, the identification of protein domains (for example from the Pfam database) in these organisms can be difficult, and important domains are easily missed. A computational approach that improves the sensitivity of Pfam domain detection significantly extended the Pfam domain coverage of all selected genomes, by proposing new occurrences of domains as well as new domain families that have never been reported earlier.

Oliviu Radulescu ("Dynamique des Interactions Membranaires Normales et Pathologiques", UMR5235, CNRS-UM2) uses systems biology with a similar general objective and presented his methodology for building kinetic integrated models of complex processes typical of these pathogens. He illustrated his approach with the phospholipid metabolism of Plasmodium falciparum, which is essential to the parasite for synthesizing considerable amounts of membrane. The distribution and kinetics of metabolic fluxes in the different pathways, integrating transcriptome and proteome data, should help predicting the diverse alternative pathways that may be used by the parasite in the presence or absence of a drug.

In a more concrete approach, Maryse Lebrun ("Dynamique des Interactions Membranaires Normales et Pathologiques", UMR5235, CNRS-UM2) reported the recent achievements of her group about the mechanisms of host cell invasion in Apicomplexan parasites. Being obligatory intracellular organisms, Apicomplexa have developed a unique invasion mechanism that is conserved across the phylum involving a tight interaction formed between the host cell and the parasite surfaces called Moving Junction (MJ). Continuing her success story about rhoptry proteins, she showed how specific protein interactions are important for the invasive process both in Toxoplasma and Plasmodium. Peptides derived from the ectodomain of RON2 were able to compete in the nanomolar range with the native 
RON2 for AMA1 interaction, opening the route to designing low molecular weight drugs capable of disrupting parasite invasion.

Pursuing the Henri Vial group's efforts towards designing successful new inhibitors of the membrane phospholipids metabolism of Plasmodium falciparum, Rachel Cerdan ("Dynamique des Interactions Membranaires Normales et Pathologiques", UMR5235, CNRS-UM2) reported the recent elucidation of phosphatidylcholine (PC) and phosphatidylethanolamine (PE) biosynthesis. Focusing on the two cytidylyltransferases (CCT and ECT) enzymes of the corresponding metabolic pathways, they established their kinetic properties, as well as three-dimensional models, to describe the substrate binding sites and the specificities of the catalytic domains - thereby opening perspectives to their inhibition.

In the same field and closer to drug development, Suzanne Peyrottes ("Institut des Biomolécules Max Mousseron", UMR 5247 CNRS-UM1-UM2), presented new analogues of Albitiazolium (T3/SAR97276) as potential anti-malarial agents. Using a prodrug approach allowed improving the absolute oral bioavailability of T3. T3 analogs were then efficiently synthesized and two promising compounds incorporating modified linkers were able to cure $P$. vinckei malarial infection at very low doses in mice.

Finally, Jean-François Hernandez ("Institut des Biomolécules Max Mousseron", UMR 5247 CNRS-UM1-UM2) described a novel potential drug target for the treatment of diseases due to protozoa, that his and other groups in Montpellier are investigating using Leishmania and Trypanosoma as models : HslVU is an ancestral form of the $26 \mathrm{~S}$ proteasome present in the mitochondrion of these parasites and essential to cell survival. An in vitro test for the activity of the protease was developed and peptides designed to inhibit the assembly of the HslVU complex, with the help of molecular modelling aiming at establishing structural requirements, which could lead to high affinity and stable ligand analogues. This multidisciplinary project, associating chemists (J.-F. Hernandez), biochemists and structural biologists ((Olivier Coux's group and Andrey Kajava, respectively, "Centre de Recherches en Biologie Moléculaire", UMR5237 CNRS-UM1-UM2) and molecular parasitologists (Patrick Bastien's group, "MIVEGEC", UMR 5290 CNRS-224 IRD-UM1-UM2) is emblematic of the strength and dynamics of this research field in Montpellier.

\section{Satellite Symposium : Setting up a regional network of Biosafety level 3 (BSL3) facilities} Nathalie Chazal (CNRS, UMR 5236, UM1,UM2), Chair

With more than 70,000 students, its 6,000 researchers working in public or private organizations and its many innovative start up and major private companies in the biomedical field, Montpellier city and more generally the Languedoc-Roussillon area, is a major think tank in the French biology and health researches. In this context, the Languedoc-Roussillon area is a global reference center in research on infectious diseases that includes nearly 100 people in hospital structures (CHU and CRLC), more than 500 scientists from Universities and National research institutions (CNRS, INSERM, IRD, CIRAD, INRA, CEA). The research covers the full range of basic and applied sciences and thus encompasses both the cellular and molecular biology of infectious processes. The research teams working on 
infectious pathogens and / or pathogen vectors, are bridging the gap between biologists, biophysicists and chemists for drug discovery.

Recently, the international visibility of Montpellier Infectious Diseases was reinforced by the development of high level biosafety laboratories (BSL) and platforms (it includes BSL3, such as the large BSL3 facility "CEMIPAI ", as well as animals and insects facilities classified at level 3 (A3, I3), such as the I3 facility " vectopôle "), placing Montpellier and more generally the Languedoc-Roussillon area among the leaders at the national level in terms of BSL3 infrastructures.

In this context, the creation of a regional network bringing together BSL3, A3, and I3 facilities has emerged. The ambition of the network is to facilitate and develop exchanges and collaborations between Montpellier' class 3 platforms and other regional sites promoting complementary tools and techniques, providing expertise in $R \& D$ and industrial relations, increasing visibility towards funders monitor and ensuring updates (regulatory monitoring, technological, harmonization of methods). The network will also develop a range of training for work in BSL3 facilities thereby allowing answer the major challenges regarding infectious diseases.

About 50 participants involved in the use and/or management of BSL3, A3 and I3 facilities attended the first satellite symposium of the Regional network of BSL3 facilities. N.Chazal (CPBS, CNRS UM2-UM2) provided an overview of the major position of Montpellier in the field of infectious diseases and more generally of Languedoc-Roussillon area and proceed to an inventory of BSL3 regional facilities. J-D Arnaud (ECE-A3L3, UMS3426,UM2) proposed a definition of the regional BSL3/A3/I3 network and presented a proposal for the governance and management of the network. M-T Alvarez (ECE-A3L3, UMS3426,UM2) reported specific examples of acute and recurrent issues related to the used of the BSL3 facilities (training, transport of infectious material, external emergency plans, risk management, policy). R. Orti (IGH-IGMM-IGF-CRBM) finally pointed the necessity to share and harmonize procedures using, as an example, the problem of bench decontamination methods. At the end of the symposium, it was decided to establish working groups within the network. Each working group will met regularly and will be in charge to address specific questions which would benefit from a brainstorming approach. Each year two meetings will be organized to bring together all the players in the network to coordinate all actions incurred.

\section{Acknowledgments:}

The organizing committee of the MID' 2nd annual meeting thank the Languedoc Roussillon local authorites (Région LR), Universities of Montpellier and national research institutions, Biocampus Montpellier, Idenix pharmaceuticals, Dominique Dutscher, StarLab, Biohit, Oxeltis, Copyshop and Elsevier-MEEGID for their support and/or sponsoring of this meeting.

Session chair and organizing committee (in alphabetic order): Bastien P., Braun-Breton C., Chazal N., Devaux C., Foulongne V., Frank M., Hernandez J.F., Kremer L., Lebrun M., Le Moing V., Leonetti J.P., Lionne C., Peyrottes S. 
Correspondence: Christian Devaux, Centre d'études d'agents pathogènes et biotechnologies pour la santé (CPBS), UMR5236, CNRS, UM1, UM2, 1919 Route de Mende, 34293, Montpellier Cedex 5, France ; email : christian.devaux@cpbs.cnrs.fr 